\title{
Theoretical study of lesion detectability of MAP reconstruction using computer observers
}

\author{
Jinyi Qi and Ronald H. Huesman \\ Center for Functional Imaging, Lawrence Berkeley National Laboratory \\ One Cyclotron Road, MS 55-121, Berkeley, CA 94720 \\ TEL: (510) 486-4695, FAX: (510) 486-4768 \\ Email: jqi@lbl.gov
}

\begin{abstract}
This work was supported by the U.S. Department of Health and Human Services, National Heart, Lung and Blood Institute under grant P01 HL25840, by the National Cancer Institute under grant R01 CA 59794, and by the Director, Office of Science, Office of Biological and Environmental Research, Medical Sciences Division, of the U.S. Department of Energy under contract no. DE-AC03-76SF00098.
\end{abstract}




\begin{abstract}
The low signal-to-noise ratio in emission data has stimulated the development of statistical image reconstruction methods based on the maximum a posteriori (MAP) principle. Experimental examples have shown that statistical methods improve image quality compared to the conventional filtered backprojection (FBP) method. However, these results depend on isolated data sets. Here we study the lesion detectability of MAP reconstruction theoretically, using computer observers. These theoretical results can be applied to different object structures. They show that for a quadratic smoothing prior, the lesion detectability using the prewhitening observer is independent of the smoothing parameter and the neighborhood of the prior, while the non-prewhitening observer exhibits an optimum smoothing point. We also compare the results to those of FBP reconstruction. The comparison shows that for ideal positron emission tomography (PET) systems (where data are true line integrals of the tracer distribution) the MAP reconstruction has a higher signal-to-noise ratio for lesion detection than FBP reconstruction due to the modeling of the Poisson noise. For realistic systems, MAP reconstruction further benefits from accurately modeling the physical photon detection process in PET.
\end{abstract}

\title{
Keywords
}

Positron emission tomography, MAP estimation, lesion detection, computer observer.

\section{INTRODUCTION}

Statistical image reconstruction methods based on the maximum a posteriori (MAP) principle (or maximizing a penalized likelihood function) have been developed for positron emission tomography (PET) to improve image quality [1], [2], [3], [4]. While most PET data are currently reconstructed using the filtered backprojection (FBP) algorithm, accuracy of the reconstructed images is limited by the approximations implicit in the line integral model on which the reconstruction formulae are based. In contrast, statistical methods can adopt arbitrarily accurate models for the mapping between the source volume and the acquired data (sinograms). The second limitation of the analytic approaches is that they do not take account of the statistical variability inherent in photon-limited coincidence detection. The resulting noise in FBP reconstructions is controlled, at the expense of resolution, by varying the cut-off frequency of a linear filter applied to the sinogram. Since the noise is signal dependent, this type of filtering is not particularly effective at achieving a good bias-variance trade-off. In contrast, statistical approaches explicitly model the noise associated with photon-limited data.

Experimental results have shown that MAP reconstruction can achieve a better resolution and variance trade-off compared to the FBP method. However, these results depend on isolated data sets, and the resolution and variance curve may not reflect the outcome of clinical applications of PET. In this paper we 
theoretically study the benefit of using MAP reconstruction in one of the major applications of clinical PET: lesion detection [5], [6]. A standard methodology for comparing lesion detectability is the receiver operating characteristic (ROC) study that compares true positive vs. false positive rates for human observers for the task of lesion detection in images reconstructed using two or more different methods [7], [8], [9]. However, the human observer ROC study is extremely time-consuming. To overcome this problem, computer observers based on signal detection theory have been developed. Computer observers allow fast evaluation of different algorithms and also provide the possibility of theoretical analysis of lesion detectability. There is a substantial body of literature dealing with the development of computer observers with comparison to human observers, and using them in ROC studies [10], [11], [12], [13], [14], [15], [16], [17], [18], [19], [20], [21]. Research is active in the search for better computer observer models that match human performance in a wide range of situations. Here we use two typical linear computer observers, the prewhitening observer and the non-prewhitening observer, to theoretically analyze the lesion detectability of MAP reconstructions. The results not only provide a theoretical proof of the improvement in lesion detection using MAP reconstruction, but will be useful for optimization of MAP reconstruction algorithms ${ }^{1}$ and PET system design [22].

The paper is organized as follows. The formulations of MAP reconstruction and computer observers are described in Section II. In Section III, we derive the theoretical expressions for lesion detectability of MAP reconstructions. For comparison, the theoretical results are also derived for FBP reconstruction in Section IV. The improvements in lesion detection using MAP reconstruction compared to FBP are shown in Section V. Finally, conclusions and discussions are presented in Section VI.

\section{BACKGROUND}

\section{A. MAP Reconstruction}

PET data are well modeled as a collection of independent Poisson random variables, and the appropriate log-likelihood function is given by

$$
L(\boldsymbol{y} \mid \boldsymbol{x})=\sum_{i}\left(y_{i} \log \bar{y}_{i}-\bar{y}_{i}-\log y_{i} !\right)
$$

where $\boldsymbol{x} \in \mathbb{R}^{N \times 1}$ is the unknown image, $\boldsymbol{y} \in \mathbb{R}^{M \times 1}$ the measured sinogram, and $\overline{\boldsymbol{y}} \in \mathbb{R}^{M \times 1}$ the mean of the sinogram. The mean sinogram, $\overline{\boldsymbol{y}}$, is related to the image, $\boldsymbol{x}$, through an affine transform

$$
\overline{\boldsymbol{y}}=\boldsymbol{P} \boldsymbol{x}+\boldsymbol{s}+\boldsymbol{r},
$$

${ }^{1}$ Note that when the prior term is made data-adaptive, the algorithm ceases to be a true Bayesian method. 
where $\boldsymbol{P} \in \mathbb{R}^{M \times N}$ is the detection probability matrix, and $s \in \mathbb{R}^{M \times 1}$ and $\boldsymbol{r} \in \mathbb{R}^{M \times 1}$ account for the presence of scatter and randoms in the data, respectively.

The detection probability matrix $\boldsymbol{P}$ can be modeled using a factored matrix approach

$$
\boldsymbol{P}=\boldsymbol{P}_{\mathrm{sens}} \boldsymbol{P}_{\text {attn }} \boldsymbol{P}_{\mathrm{sys}}
$$

where $\boldsymbol{P}_{\text {sys }}$ is a projection matrix determined by the PET system, with each element $(i, j)$ equal to the probability of a photon pair produced in voxel $j$ being detected by the detector pair $i$ in the absence of attenuation and assuming uniform detector sensitivity. $\boldsymbol{P}_{\mathrm{sys}}$ can be factored further as a product of a geometric projection matrix and a sinogram blurring matrix to efficiently model the depth dependent geometric sensitivity and detector response as proposed in [23], [24]. $\boldsymbol{P}_{\text {attn }}$ and $\boldsymbol{P}_{\text {sens }}$ are diagonal matrices containing the attenuation factors and normalization factors, respectively.

Most image priors in PET image reconstruction use a Gibbs distribution of the form

$$
\frac{1}{Z} e^{-\beta U(\boldsymbol{x})},
$$

where $U(\boldsymbol{x})$ is the energy function, $\beta$ is the smoothing parameter that controls the resolution of the reconstructed image, and $Z$ is a normalization constant. Here we focus on the widely used quadratic priors, for which the energy function can be expressed as

$$
U(\boldsymbol{x})=\frac{1}{2} \boldsymbol{x}^{\prime} \boldsymbol{R} \boldsymbol{x},
$$

where $\boldsymbol{R}$ is a positive definite (or semidefinite ${ }^{2}$ ) matrix. Pair-wise membrane priors and thin-plate priors are special cases of (5).

Combining the likelihood function and the image prior, the MAP reconstruction is found as

$$
\hat{\boldsymbol{x}}(\boldsymbol{y})=\arg \max _{\boldsymbol{x} \geq 0}[L(\boldsymbol{y} \mid \boldsymbol{x})-\beta U(\boldsymbol{x})] .
$$

Since $L(\boldsymbol{y} \mid \boldsymbol{x})$ is a concave function of $\boldsymbol{x},(6)$ generally has a unique solution for convex priors.

\section{B. Lesion Detection}

Detecting a cancerous lesion in a PET image involves searching for an abnormal "hot spot" in a known background. The performance of the detection is often measured by ROC. Here we use a "signal-knownexactly, background-known-exactly" (SKE-BKE) computer observer ROC to study the lesion detectability of MAP and FBP reconstruction. The computer observers that we used are the prewhitening (PW) observer and the non-prewhitening (NPW) observer [10], [11].

\footnotetext{
${ }^{2}$ Strictly speaking, when $\boldsymbol{R}$ is semidefinite, the normalization constant $Z=\infty$.
} 
Both observers compute a linear test statistic, $\eta(\hat{\boldsymbol{x}})$, from the reconstructed image $\hat{\boldsymbol{x}}$. The PW observer corresponds to a likelihood ratio test on a Gaussian distributed image for which the covariance matrix is known. The NPW observer is similar but does not use the covariance matrix. The decision whether there is a lesion or not is then made by comparing this statistic to a preselected threshold. If $\eta(\hat{\boldsymbol{x}})$ exceeds the decision threshold, $\hat{\boldsymbol{x}}$ is determined to have a lesion, otherwise it is not.

Let $\boldsymbol{f}_{l}$ be the lesion profile and $\boldsymbol{f}_{0}$ the background image. As we are particularly interested in detection of small lesions, we can assume the lesion is so small that it hardly changes the noise in the data. Thus, the noise in $\hat{\boldsymbol{x}}$ is independent of the presence of the lesion. Let $\boldsymbol{h}\left(\boldsymbol{f}_{0}+\boldsymbol{f}_{l}\right)$ and $\boldsymbol{h}\left(\boldsymbol{f}_{0}\right)$ denote the mean reconstructions of the image with and without the lesion present, respectively. Then the test statistic of the PW observer is

$$
\eta_{\mathrm{PW}}(\hat{\boldsymbol{x}})=\frac{\left[\boldsymbol{h}\left(\boldsymbol{f}_{0}+\boldsymbol{f}_{l}\right)-\boldsymbol{h}\left(\boldsymbol{f}_{0}\right)\right]^{\prime} \Sigma^{-1}\left[\hat{\boldsymbol{x}}-\boldsymbol{h}\left(\boldsymbol{f}_{0}\right)\right]}{\left[\boldsymbol{h}\left(\boldsymbol{f}_{0}+\boldsymbol{f}_{l}\right)-\boldsymbol{h}\left(\boldsymbol{f}_{0}\right)\right]^{\prime} \Sigma^{-1}\left[\boldsymbol{h}\left(\boldsymbol{f}_{0}+\boldsymbol{f}_{l}\right)-\boldsymbol{h}\left(\boldsymbol{f}_{0}\right)\right]}=\frac{\boldsymbol{z}^{\prime} \Sigma^{-1}\left[\hat{\boldsymbol{x}}-\boldsymbol{h}\left(\boldsymbol{f}_{0}\right)\right]}{\boldsymbol{z}^{\prime} \Sigma^{-1} \boldsymbol{z}},
$$

where $\Sigma$ is the covariance matrix of $\hat{x}$ and

$$
z \equiv \boldsymbol{h}\left(\boldsymbol{f}_{0}+\boldsymbol{f}_{l}\right)-\boldsymbol{h}\left(\boldsymbol{f}_{0}\right)
$$

is the observer template. The test statistic, $\eta_{\mathrm{PW}}(\hat{\boldsymbol{x}})$, has been normalized such that it is null when $\hat{\boldsymbol{x}}=$ $\boldsymbol{h}\left(\boldsymbol{f}_{0}\right)$ and is unity when $\hat{\boldsymbol{x}}=\boldsymbol{h}\left(\boldsymbol{f}_{0}+\boldsymbol{f}_{l}\right)$, but it is not restricted to this range of values. The observer detection performance can be measured by the signal-to-noise ratio (SNR) of $\eta_{\mathrm{PW}}(\hat{\boldsymbol{x}})$

$$
\mathrm{SNR}^{2}\left[\eta_{\mathrm{PW}}(\hat{\boldsymbol{x}})\right]=\frac{\left\{\eta_{\mathrm{PW}}\left[\boldsymbol{h}\left(\boldsymbol{f}_{0}+\boldsymbol{f}_{1}\right)\right]-\eta_{\mathrm{PW}}\left[\boldsymbol{h}\left(\boldsymbol{f}_{0}\right)\right]\right\}^{2}}{\operatorname{var}\left[\eta_{\mathrm{PW}}(\hat{\boldsymbol{x}})\right]}=\frac{1}{\operatorname{var}\left[\eta_{\mathrm{PW}}(\hat{\boldsymbol{x}})\right]}=\boldsymbol{z}^{\prime} \Sigma^{-1} \boldsymbol{z} .
$$

When $\eta(\hat{\boldsymbol{x}})$ is normally distributed, the SNR is related to the area under the ROC curve (AUC) by [11]

$$
\mathrm{AUC}=\frac{1}{2}\left[1+\operatorname{erf}\left(\frac{\mathrm{SNR}}{2}\right)\right]
$$

where $\operatorname{erf}(x)$ is the error function.

The NPW observer uses a simple matched filter to compute the test statistic

$$
\eta_{\mathrm{NPW}}(\hat{\boldsymbol{x}})=\frac{\boldsymbol{z}^{\prime}\left[\hat{\boldsymbol{x}}-\boldsymbol{h}\left(\boldsymbol{f}_{0}\right)\right]}{\boldsymbol{z}^{\prime} \boldsymbol{z}} .
$$

Eq.(10) differs from (7) only by deletion of $\Sigma^{-1}$ that accounts for the prewhitening operation. It is also normalized to have the values of unity and null for mean images with and without lesion present, respectively. The SNR of the NPW observer is given by

$$
\mathrm{SNR}^{2}\left[\eta_{\mathrm{NPW}}(\hat{\boldsymbol{x}})\right]=\frac{1}{\operatorname{var}\left[\eta_{\mathrm{NPW}}(\hat{\boldsymbol{x}})\right]}=\frac{\left(\boldsymbol{z}^{\prime} \boldsymbol{z}\right)^{2}}{\boldsymbol{z}^{\prime} \Sigma \boldsymbol{z}} .
$$


It is easy to show that $\mathrm{SNR}_{\mathrm{NPW}} \leq \mathrm{SNR}_{\mathrm{PW}}$ for any $z$ and $\Sigma$, where equality is achieved if and only if $\Sigma=I$ (up to a scaling factor). This indicates that the NPW observer is inferior to the PW observer in terms of detecting lesions. In fact, with Gaussian noise, the PW observer gives the optimal performance for lesion detection.

It has been found that in many situations the PW observer correlates with human performance, especially when noise correlation is introduced by the randomness of signal and background [25], [26]. However, the prewhitening step does make the PW observer immune to any invertible post filtering while, on the other hand, human observers often benefit from proper post filtering. Myers et al. [27], [28] found that with bandpass or high-pass post filtering, the NPW observer and channelized prewhitening observers predict human performance better than the PW observer.

Channelized observers have gained much interest since Yao et al. [12] showed that they have good correlation with human performance under both post filtering and random background situations. Research has been conducted to compare channelized observers with human observers for various task [15], [18], [19], [20], [21]. Most papers show favorable results, but counter results also exist [19]. The relative performance of different channel functions is also dependent on the lesion being detected and the background [15], [21].

Not attempting to determine the best predictor of human performance, for sake of simplicity, we choose the PW observer and the NPW observer. Extension of the analysis to other linear observers, such as the channelized Hotelling observer [12], is relatively straightforward. In fact, the PW and NPW observers can be viewed as special cases of channelized observers: the PW observer uses the maximum number of unoverlapped channels, and the NPW observer uses only one channel with the channel response being the impulse response function of the matched filter. Hence, we expect that the performance of other channelized observers will lie between these two extremes.

Since the PW observer is immune to any invertible post filtering, we do not expect it to predict human performance; however, it will tell how much information contained in the reconstructed image can be used for lesion detection, and it will give an upper bound of lesion detectability that is achievable when all the information is used. By comparing the SNR of the PW observer on a reconstructed image with the SNR of the PW observer on the corresponding raw sinogram, one can find out whether a reconstruction algorithm throws away any useful information for lesion detection. The NPW observer will have better correlation to human performance and will show how well a human might perform the same task. 


\section{LESION DETECTABILITY OF MAP RECONSTRUCTION}

\section{A. Observer Template for MAP Reconstruction}

The MAP estimator (6) is nonlinear in the data and its properties are object dependent. Even for linear estimators such as FBP, the noise properties are still spatially variant due to the Poisson statistics in the data. Therefore, we study the lesion detectability locally assuming local stationarity in the image [29]. This assumption has been successfully used in analyzing the resolution and noise properties of 2D and 3D PET reconstructions with little degradation in accuracy [29], [30], [31].

Since the lesion is quite small, we can compute the observer template using a first order Taylor series expansion of the MAP reconstruction $\hat{\boldsymbol{x}}(\boldsymbol{y})$ at the point $\boldsymbol{y}=\overline{\boldsymbol{y}}=\boldsymbol{P} \boldsymbol{f}_{0}+\boldsymbol{s}+\boldsymbol{r}$ :

$$
\hat{\boldsymbol{x}}(\boldsymbol{y}) \approx \hat{\boldsymbol{x}}(\overline{\boldsymbol{y}})+\nabla_{y} \hat{\boldsymbol{x}}(\overline{\boldsymbol{y}})(\boldsymbol{y}-\overline{\boldsymbol{y}})
$$

This approximation has been widely used in analyzing the first and second order statistics of the maximum likelihood (ML) reconstruction and MAP reconstruction with quadratic priors [29], [30], [31], [32], [33]. As a result, we have

$$
\boldsymbol{z}_{\mathrm{MAP}}=\nabla_{y} \hat{\boldsymbol{x}}(\overline{\boldsymbol{y}}) \boldsymbol{P} \boldsymbol{f}_{l}
$$

Now we restrict our attention to the situations where the solution of (6) satisfies

$$
0=\left.\frac{\partial}{\partial x_{j}}[L(\boldsymbol{y} \mid \boldsymbol{x})-\beta U(\boldsymbol{x})]\right|_{\boldsymbol{x}=\hat{\boldsymbol{x}}(\boldsymbol{y})}, \quad j=1, \ldots, M
$$

While this assumption precludes inequality constraints, it appears to work well except for regions with very low activity. Differentiating (14) with respect to $y_{i}$ by applying the chain rule and solving the resulting equation, we get [32]

$$
\begin{aligned}
\nabla_{y} \hat{\boldsymbol{x}}(\overline{\boldsymbol{y}}) & =\left.\left\{-\left.\nabla_{x x}[L(\boldsymbol{y} \mid \boldsymbol{x})-\beta U(\boldsymbol{x})]\right|_{\boldsymbol{x}=\hat{\boldsymbol{x}}(\boldsymbol{y})}\right\}^{-1} \nabla_{x y}[L(\boldsymbol{y} \mid \boldsymbol{x})-\beta U(\boldsymbol{x})]\right|_{\boldsymbol{x}=\hat{\boldsymbol{x}}(\boldsymbol{y})} \\
& =\left\{\boldsymbol{P}^{\prime} \operatorname{diag}\left[\frac{\bar{y}_{i}}{(\boldsymbol{P} \hat{\boldsymbol{x}}+\boldsymbol{s}+\boldsymbol{r})_{i}^{2}}\right] \boldsymbol{P}+\beta \boldsymbol{R}\right\}^{-1} \boldsymbol{P}^{\prime} \operatorname{diag}\left[\frac{1}{(\boldsymbol{P} \hat{\boldsymbol{x}}+\boldsymbol{s}+\boldsymbol{r})_{i}}\right]
\end{aligned}
$$

where the $(j, k)$ th element of the operator $\nabla_{x x}$ is $\frac{\partial^{2}}{\partial x_{j} \partial x_{k}}$, the $(j, l)$ th element of the operator $\nabla_{x y}$ is $\frac{\partial^{2}}{\partial x_{j} \partial y_{l}}$, and $\operatorname{diag}\left[y_{i}\right]$ denotes a diagonal matrix with the $(i, i)$ th element equal to $y_{i}$.

In general, $\hat{\boldsymbol{x}}$ is a slightly blurred version of $\boldsymbol{x}$, so the projection $\boldsymbol{P} \hat{\boldsymbol{x}}+\boldsymbol{s}+\boldsymbol{r}$ is approximately equal to the mean of the data, $\overline{\boldsymbol{y}}$. Therefore, we can simplify the above expression to

$$
\nabla_{y} \hat{\boldsymbol{x}}(\overline{\boldsymbol{y}}) \approx[\boldsymbol{F}+\beta \boldsymbol{R}]^{-1} \boldsymbol{P}^{\prime} \operatorname{diag}\left[\frac{1}{\bar{y}_{i}}\right]
$$


where $\boldsymbol{F}=\boldsymbol{P}^{\prime} \operatorname{diag}\left[\frac{1}{\bar{y}_{i}}\right] \boldsymbol{P}$ is the Fisher information matrix. Substituting (16) into (13) results in

$$
\boldsymbol{z}_{\mathrm{MAP}} \approx[\boldsymbol{F}+\beta \boldsymbol{R}]^{-1} \boldsymbol{F} \boldsymbol{f}_{l}
$$

Let $f_{l}$ be a small lesion centered at voxel $j$. Now we use the local invariant approximation as proposed in [29] to simplify (17) to

$$
\boldsymbol{z}_{\mathrm{MAP}} \approx[\boldsymbol{F}(j)+\beta \boldsymbol{R}]^{-1} \boldsymbol{F}(j) \boldsymbol{f}_{l}
$$

where $\boldsymbol{F}(j)$ is a locally invariant approximation of $\boldsymbol{F}$ at voxel $j$, i.e., $\boldsymbol{F}(j)$ is a block Toeplitz positive semidefinite matrix with the $j$ th column approximately equal to the $j$ th column of $\boldsymbol{F}$ [29]. To further simplify the analysis, we can approximate $\boldsymbol{F}(j)$ by [29], [30], [33]

$$
\boldsymbol{F}(j) \approx \kappa_{j}^{2} \boldsymbol{S}(j)
$$

where $\boldsymbol{S}(j)$ is the local invariant approximation of $\boldsymbol{P}_{\text {sys }}^{\prime} \boldsymbol{P}_{\text {sys }}$ at voxel $j$, and $\kappa_{j}^{2}$ is

$$
\kappa_{j}^{2} \equiv \frac{1}{\sum_{i} g_{i j}^{2}} \sum_{i} \frac{g_{i j}^{2} a_{i}^{2} n_{i}^{2}}{\bar{y}_{i}},
$$

where $g_{i j}$ is the $(i, j)$ th element in $\boldsymbol{P}_{\text {sys }}, a_{i}$ and $n_{i}$ are $(i, i)$ th diagonal elements of $\boldsymbol{P}_{\text {attn }}$ and $\boldsymbol{P}_{\text {sens }}$, respectively. Here $\boldsymbol{P}_{\text {sys }}^{\prime} \boldsymbol{P}_{\text {sys }}$ is not required to be spatially invariant as in [33]. When the factored matrix is used for $\boldsymbol{P}_{\text {sys }}$ as in [23], [24], the approximation proposed in [29] (Eq. (14)) can be used in place of (20).

Since nonzero elements in $\boldsymbol{S}(j)$ and $\boldsymbol{R}(j)$ are located only around the diagonal of each block, $\boldsymbol{S}(j)$ and $\boldsymbol{R}(j)$ can be approximated by block circulant matrices, and their approximate inverses can be computed using Fourier transforms [29], [34]. Hence we can write $z_{\mathrm{MAP}}$ in the following compact form:

$$
\begin{aligned}
\boldsymbol{z}_{\mathrm{MAP}} & \approx \boldsymbol{Q}^{\prime} \boldsymbol{Q}[\boldsymbol{F}(j)+\beta \boldsymbol{R}(j)]^{-1} \boldsymbol{Q}^{\prime} \boldsymbol{Q} \boldsymbol{F}(j) \boldsymbol{Q}^{\prime} \boldsymbol{Q} \boldsymbol{f}_{l} \\
& \approx \boldsymbol{Q}^{\prime}\left[\boldsymbol{Q F}(j) \boldsymbol{Q}^{\prime}+\beta \boldsymbol{Q} \boldsymbol{R}(j) \boldsymbol{Q}^{\prime}\right]^{-1}\left[\boldsymbol{Q} \boldsymbol{F}(j) \boldsymbol{Q}^{\prime}\right] \boldsymbol{Q} \boldsymbol{f}_{l} \\
& \approx \boldsymbol{Q}^{\prime} \operatorname{diag}\left[\frac{\lambda_{i}(j)}{\lambda_{i}(j)+\beta \mu_{i} / \kappa_{j}^{2}}\right] \boldsymbol{Q} \boldsymbol{f}_{l}
\end{aligned}
$$

where $\left\{\lambda_{i}(j), i=1, \ldots, N\right\}$ is the Fourier transform of $\boldsymbol{S}(j)$ and $\left\{\mu_{i}, i=1, \ldots, N\right\}$ is the Fourier transform of $\boldsymbol{R} . \boldsymbol{Q}$ and $\boldsymbol{Q}^{\prime}$ represent the Kronecker form of the FFT and its inverse, respectively.

\section{B. Covariance Matrix of MAP Reconstruction}

When noise in the reconstruction is small compared to the mean of background, which is often the case in PET, we can obtain the following approximation of the covariance matrix of MAP reconstruction from 
(12) and (16) [32]

$$
\begin{aligned}
\Sigma(\hat{\boldsymbol{x}}) & \approx \nabla_{y} \hat{\boldsymbol{x}}(\overline{\boldsymbol{y}}) \operatorname{cov}(\boldsymbol{y})\left[\nabla_{\mathrm{y}} \hat{\boldsymbol{x}}(\overline{\boldsymbol{y}})\right]^{\prime} \\
& \approx[\boldsymbol{F}+\beta \boldsymbol{R}]^{-1} \boldsymbol{F}[\boldsymbol{F}+\beta \boldsymbol{R}]^{-1} .
\end{aligned}
$$

Using the local invariant approximation and the approach described in the previous section, we can write the covariance matrix for the $j$ th voxel as [29]

$$
\begin{aligned}
\Sigma_{j}(\hat{\boldsymbol{x}}) & \approx[\boldsymbol{F}(j)+\beta \boldsymbol{R}(j)]^{-1} \boldsymbol{F}(j)[\boldsymbol{F}(j)+\beta \boldsymbol{R}(j)]^{-1} \\
& \approx \boldsymbol{Q}^{\prime} \operatorname{diag}\left[\frac{\lambda_{i}(j) / \kappa_{j}^{2}}{\left(\lambda_{i}(j)+\beta \mu_{i} / \kappa_{j}^{2}\right)^{2}}\right] \boldsymbol{Q} .
\end{aligned}
$$

\section{SNR of MAP Reconstruction}

Substituting (21) and (23) into (9), we get the $\mathrm{SNR}_{\mathrm{PW}}$ of the PW observer for detecting lesions with MAP reconstruction:

$$
\begin{aligned}
\mathrm{SNR}_{\mathrm{PW}, \mathrm{MAP}}^{2} & =\boldsymbol{z}_{\mathrm{MAP}}^{\prime}\left[\Sigma_{j}(\hat{\boldsymbol{x}})\right]^{-1} \boldsymbol{z}_{\mathrm{MAP}} \\
& \approx \boldsymbol{f}_{l}^{\prime} \boldsymbol{Q}^{\prime} \operatorname{diag}\left[\frac{\lambda_{i}(j)}{\lambda_{i}(j)+\beta \mu_{i} / \kappa_{j}^{2}} \frac{\left(\lambda_{i}(j)+\beta \mu_{i} / \kappa_{j}^{2}\right)^{2}}{\lambda_{i}(j) / \kappa_{j}^{2}} \frac{\lambda_{i}(j)}{\lambda_{i}(j)+\beta \mu_{i} / \kappa_{j}^{2}}\right] \boldsymbol{Q} \boldsymbol{f}_{l} \\
& =\kappa_{j}^{2} \boldsymbol{f}_{l}^{\prime} \boldsymbol{Q}^{\prime} \operatorname{diag}\left[\lambda_{i}(j)\right] \boldsymbol{Q} \boldsymbol{f}_{l} \\
& =\kappa_{j}^{2} \boldsymbol{f}_{l}^{\prime} \boldsymbol{P}_{\mathrm{sys}}^{\prime} \boldsymbol{P}_{\mathrm{sys}} \boldsymbol{f}_{l} .
\end{aligned}
$$

Note that (24) shows that $\mathrm{SNR}_{\mathrm{PW}}$ is independent of the quadratic prior function $\boldsymbol{R}$ and the smoothing parameter $\beta$ and is equal to that of the ML solution. This is because the quadratic prior function does not differentiate changes in signal and noise, and they are equally penalized. Hence, using the quadratic prior function does not add any information to the reconstruction. Within the accuracy of the above approximations, $\mathrm{SNR}_{\mathrm{PW}, \mathrm{MAP}}$ is equal to the $\mathrm{SNR}$ of the $\mathrm{PW}$ observer applied directly in the sinogram domain. This indicates that MAP reconstruction retains all information in the data.

Similarly, we can get $\mathrm{SNR}_{\mathrm{NPW}}$ for the NPW observer by substituting (21) and (23) into (11):

$$
\begin{aligned}
\mathrm{SNR}_{\mathrm{NPW}, \mathrm{MAP}}^{2}= & \frac{\left(\boldsymbol{z}_{\mathrm{MAP}}^{\prime} \boldsymbol{z}_{\mathrm{MAP}}\right)^{2}}{\boldsymbol{z}_{\mathrm{MAP}}^{\prime} \Sigma_{j}(\hat{\boldsymbol{x}}) \boldsymbol{z}_{\mathrm{MAP}}} \\
\approx & \left(\boldsymbol{f}_{l}^{\prime} \boldsymbol{Q}^{\prime} \operatorname{diag}\left[\left(\frac{\lambda_{i}(j)}{\lambda_{i}(j)+\beta \mu_{i} / \kappa_{j}^{2}}\right)^{2}\right] \boldsymbol{Q} \boldsymbol{f}_{l}\right)^{2} \\
& \quad \times\left(\frac{1}{\kappa_{j}^{2}} \boldsymbol{f}_{l}^{\prime} \boldsymbol{Q}^{\prime} \operatorname{diag}\left[\frac{\lambda_{i}^{3}(j)}{\left(\lambda_{i}(j)+\beta \mu_{i} / \kappa_{j}^{2}\right)^{4}}\right] \boldsymbol{Q} \boldsymbol{f}_{l}\right)^{-1} \\
= & \kappa_{j}^{2}\left(\sum_{i}\left(\frac{\lambda_{i}(j) \zeta_{i}}{\lambda_{i}(j)+\beta \mu_{i} / \kappa_{j}^{2}}\right)^{2}\right)^{2}\left(N \sum_{i} \frac{\lambda_{i}^{3}(j) \zeta_{i}^{2}}{\left(\lambda_{i}(j)+\beta \mu_{i} / \kappa_{j}^{2}\right)^{4}}\right)^{-1},
\end{aligned}
$$


where $\left\{\zeta_{i}, i=1, \ldots, N\right\}$ is the Fourier transform of the lesion image $\boldsymbol{f}_{l}$.

Unlike $\mathrm{SNR}_{\mathrm{PW}, \mathrm{MAP}}$ for the PW observer shown in (24), the NPW observer $\mathrm{SNR}_{\mathrm{NPW}, \mathrm{MAP}}$ is a function of the smoothing parameter $\beta$ and the prior neighborhood, which is consistent with human experience. (See Fig. 3 for an example of a curve of (25).) Both (24) and (25) show that the lesion detectability is spatially variant and dependent on the uncertainty information at the lesion location. The more information we have at the location (larger $\kappa_{j}$ ), the higher the detectability.

\section{LESION DETECTABILITY OF FBP RECONSTRUCTION}

\section{A. Observer Template and Covariance Matrix}

For comparison purposes we will derive SNR for lesion detection using FBP reconstructions in this section. FBP reconstruction can be written as

$$
\boldsymbol{x}_{\mathrm{FBP}}=\boldsymbol{F}_{I} \boldsymbol{B} \boldsymbol{P}_{\mathrm{sens}}^{-1} \boldsymbol{P}_{\mathrm{attn}}^{-1} \boldsymbol{y}
$$

where $\boldsymbol{F}_{I}$ is the image space reconstruction filter and $\boldsymbol{B}$ is the backprojection matrix for line integral operator. In general, $\boldsymbol{B}$ is not equal to $\boldsymbol{P}_{\text {sys }}^{\prime}$ for real PET systems because of solid angle and detector blurring effects.

Using linear system theory, the observer template for FBP reconstruction is

$$
\boldsymbol{z}_{\mathrm{FBP}}=\boldsymbol{F}_{I} \boldsymbol{B} \boldsymbol{P}_{\mathrm{sys}} \boldsymbol{f}_{l}
$$

and the covariance matrix is

$$
\begin{aligned}
\Sigma_{\mathrm{FBP}} & =\boldsymbol{F}_{I} \boldsymbol{B} \boldsymbol{P}_{\mathrm{sens}}^{-1} \boldsymbol{P}_{\mathrm{attn}}^{-1} \operatorname{cov}(\boldsymbol{y}) \boldsymbol{P}_{\mathrm{attn}}^{-1} \boldsymbol{P}_{\mathrm{sens}}^{-1} \boldsymbol{B}^{\prime} \boldsymbol{F}_{\mathrm{I}}^{\prime} \\
& =\boldsymbol{F}_{I} \boldsymbol{B} \operatorname{diag}\left[\frac{\bar{y}_{i}}{a_{i}^{2} n_{i}^{2}}\right] \boldsymbol{B}^{\prime} \boldsymbol{F}_{I}^{\prime} .
\end{aligned}
$$

Eq. (28) shows that even though FBP is a linear estimator, the covariance is still spatially variant. To compute the detectability of the lesion at voxel $j$, we will simplify the covariance matrix for the $j$ th voxel using the local invariant approximation. Let $\boldsymbol{H}(j)$ denote the local invariant approximation of

$$
\boldsymbol{B} \operatorname{diag}\left[\frac{\bar{y}_{i}}{a_{i}^{2} n_{i}^{2}}\right] \boldsymbol{B}^{\prime}
$$

at voxel $j$. As in (19) we approximate $\boldsymbol{H}(j)$ by

$$
\boldsymbol{H}(j) \approx \xi_{j}^{2} \boldsymbol{B} \boldsymbol{B}^{\prime}
$$


where

$$
\xi_{j}^{2} \equiv \frac{1}{\sum_{i} b_{j i}^{2}} \sum_{i} \frac{b_{j i}^{2} \bar{y}_{i}}{a_{i}^{2} n_{i}^{2}} .
$$

Then the covariance matrix at voxel $j$ can be approximated by

$$
\Sigma_{\mathrm{FBP}}(j) \approx \xi_{j}^{2} \boldsymbol{F}_{I} \boldsymbol{B} \boldsymbol{B}^{\prime} \boldsymbol{F}_{I}^{\prime}
$$

This form is similar to the approximations used in [35], [36].

\section{B. SNR of FBP Reconstruction}

Substituting (27) and (31) into (9), we get the SNR of the PW observer for detecting lesions in FBP reconstruction:

$$
\begin{aligned}
\mathrm{SNR}_{\mathrm{PW}, \mathrm{FBP}}^{2} & \approx \frac{1}{\xi_{j}^{2}} \boldsymbol{f}_{l}^{\prime} \boldsymbol{P}_{\mathrm{sys}}^{\prime} \boldsymbol{B}^{\prime} \boldsymbol{F}_{I}^{\prime}\left(\boldsymbol{F}_{I} \boldsymbol{B} \boldsymbol{B}^{\prime} \boldsymbol{F}_{I}^{\prime}\right)^{-1} \boldsymbol{F}_{I} \boldsymbol{B} \boldsymbol{P}_{\mathrm{sys}} \boldsymbol{f}_{l} \\
& =\frac{1}{\xi_{j}^{2}} \boldsymbol{f}_{l}^{\prime} \boldsymbol{P}_{\mathrm{sys}}^{\prime} \boldsymbol{B}^{\prime}\left(\boldsymbol{B} \boldsymbol{B}^{\prime}\right)^{-1} \boldsymbol{B} \boldsymbol{P}_{\mathrm{sys}} \boldsymbol{f}_{l}
\end{aligned}
$$

Here we assumed the filter function $\boldsymbol{F}_{I}$ is invertible. Similar to $\mathrm{SNR}_{\mathrm{PW}, \mathrm{MAP}}, \mathrm{SNR}_{\mathrm{PW}, \mathrm{FBP}}$ is also independent of the smoothing used in reconstruction. The above result is different from that shown in [17] where noise was assumed to be stationary, which is generally not the case in PET/SPECT reconstructions.

The SNR of the NPW observer for FBP reconstruction can also be computed by

$$
\begin{aligned}
\mathrm{SNR}_{\mathrm{NPW}, \mathrm{FBP}}^{2} & \approx \frac{\left(\boldsymbol{f}_{l}^{\prime} \boldsymbol{P}_{\mathrm{sys}}^{\prime} \boldsymbol{B}^{\prime} \boldsymbol{F}_{I}^{\prime} \boldsymbol{F}_{I} \boldsymbol{B} \boldsymbol{P}_{\mathrm{sys}} \boldsymbol{f}_{l}\right)^{2}}{\xi_{j}^{2} \boldsymbol{f}_{l}^{\prime} \boldsymbol{P}_{\mathrm{sys}}^{\prime} \boldsymbol{B}^{\prime} \boldsymbol{F}_{I}^{\prime} \boldsymbol{F}_{I} \boldsymbol{B} \boldsymbol{B}^{\prime} \boldsymbol{F}_{I}^{\prime} \boldsymbol{F}_{I} \boldsymbol{B} \boldsymbol{P}_{\mathrm{sys}} \boldsymbol{f}_{l}} \\
& =\frac{1}{\xi_{j}^{2}} \frac{\left[\sum_{i} w_{i}^{2} \zeta_{i}^{2} \tilde{\lambda}_{i}^{2}(j)\right]^{2}}{N \sum_{i} w_{i}^{4} \tau_{i} \zeta_{i}^{2} \tilde{\lambda}_{i}^{2}(j)},
\end{aligned}
$$

where $\left\{w_{i}, i=1, \ldots, N\right\}$ is the Fourier transform of $\boldsymbol{F}_{I}$ (i.e., the filter frequency coefficients), $\left\{\tau_{i}, i=\right.$ $1, \ldots, N\}$ the Fourier transform of $\boldsymbol{B} \boldsymbol{B}^{\prime}$ (approximately $1 / r$ response), and $\left\{\tilde{\lambda}_{i}(j), i=1, \ldots, N\right\}$ the Fourier transform of the local invariant approximation of $\boldsymbol{B} \boldsymbol{P}_{\text {sys }}$ at voxel $j$. When $\boldsymbol{B}^{\prime}=\boldsymbol{P}$, we have $\tilde{\lambda}_{i}(j)=\lambda_{i}(j)$.

\section{IMPROVEMENTS In LESION DETECTION USING MAP RECONSTRUCTION}

\section{A. Ideal PET System with PW Observer}

Suppose we have an ideal PET system, where mean data are perfect line integrals of source distribution $\left(\boldsymbol{B}=\boldsymbol{P}_{\mathrm{sys}}^{\prime}\right)$. With this assumption (32) reduces to

$$
\mathrm{SNR}_{\mathrm{PW}, \mathrm{FBP}}^{2} \approx \frac{1}{\xi_{j}^{2}} \boldsymbol{f}_{l}^{\prime} \boldsymbol{P}_{\mathrm{sys}}^{\prime} \boldsymbol{P}_{\mathrm{sys}} \boldsymbol{f}_{l} .
$$




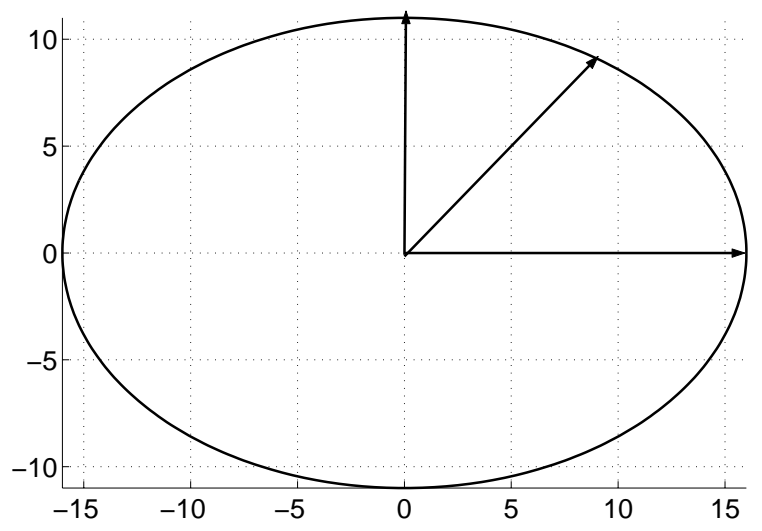

Fig. 1. Ellipse phantom with uniform activity and attenuation coefficient. The point $(0,0)$ corresponds to the radial center of the detector ring, and the labels are all in centimeter.

In this case the improvement in detectability using MAP reconstruction compared to the FBP method is

$$
\begin{aligned}
\frac{\mathrm{SNR}_{\mathrm{PW}, \mathrm{MAP}}^{2}}{\mathrm{SNR}_{\mathrm{PW}, \mathrm{FBP}}^{2}} & \approx \kappa_{j}^{2} \xi_{j}^{2}=\frac{1}{\left(\sum_{i} g_{i j}^{2}\right)^{2}} \sum_{i} \frac{g_{i j}^{2} a_{i}^{2} n_{i}^{2}}{\bar{y}_{i}} \sum_{i} \frac{g_{i j}^{2} \bar{y}_{i}}{a_{i}^{2} n_{i}^{2}} \\
& \geq \frac{1}{\left(\sum_{i} g_{i j}^{2}\right)^{2}}\left(\sum_{i} \sqrt{\frac{g_{i j}^{2} a_{i}^{2} n_{i}^{2}}{\bar{y}_{i}}} \sqrt{\frac{g_{i j}^{2} \bar{y}_{i}}{a_{i}^{2} n_{i}^{2}}}\right)^{2}=1,
\end{aligned}
$$

where equality is achieved if and only if $\bar{y}_{1} /\left(a_{1}^{2} n_{1}^{2}\right)=\bar{y}_{2} /\left(a_{2}^{2} n_{2}^{2}\right)=\cdots=\bar{y}_{N} /\left(a_{N}^{2} n_{N}^{2}\right)$.

This shows that even for an ideal system, where the PET data are truly line integrals of the source distribution, the MAP algorithm can still improve the small lesion detectability due to the modeling of the Poisson statistics of the data. The improvement as shown in (35) is independent of the count level of the data $\sum_{i} \bar{y}_{i}$ (there is a general misunderstanding that when the count level increases, the benefit of MAP reconstruction decreases), the lesion profile $\boldsymbol{f}_{l}$ (provided it is small), and the sensitivity of this voxel $\sum_{i} g_{i j}^{2}$. It is dependent on the variation in the elements $\bar{y}_{i} /\left(a_{i}^{2} n_{i}^{2}\right)$ that contribute to this particular voxel. The larger the variation, the greater the benefit of using the MAP reconstruction.

We conducted a computer simulation to show the improvement in lesion detectability for an ideal system. We used a uniform activity ellipse phantom as the background (Fig. 1). The attenuation factors, $a_{i}$, were calculated by assigning a uniform attenuation coefficient of $0.095 \mathrm{~cm}^{-1}$ inside the phantom, and detector sensitivities, $n_{i}$, were 1.0. The data were generated by computing line integrals through the phantom. As we saw above, the ratio in (35) is independent of the absolute activity level.

We computed the ratio of (35) for the points on the $x$-axis, $y$-axis, and diagonal $x=y$, as shown in Fig. 1. The results in Fig. 2 show that for this uniform ellipse phantom, the average improvement in lesion 


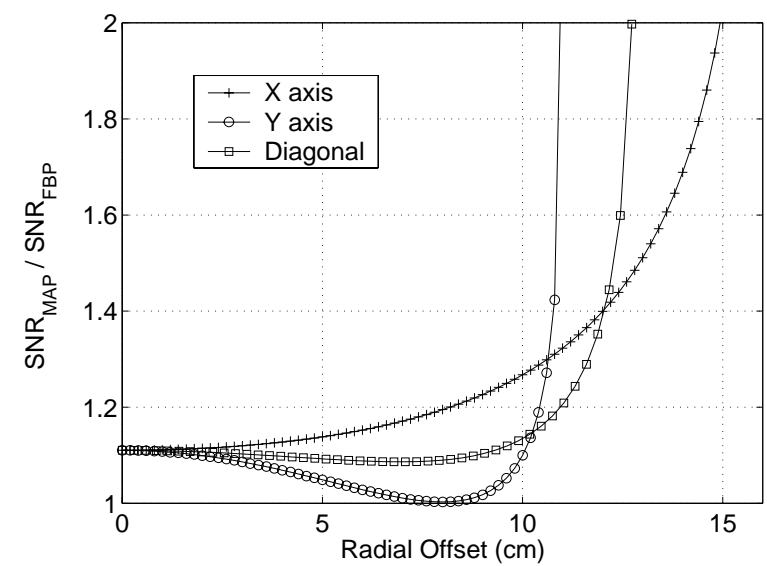

Fig. 2. Ratio of $\mathrm{SNR}_{\mathrm{MAP}}$ over $\mathrm{SNR}_{\mathrm{FBP}}$ for the voxels on the $x$-axis, $y$-axis, and diagonal $x=y$.

detection that resulted from modeling the Poisson statistics in the data is about $10 \%$ for voxels within $10 \mathrm{~cm}$ radial offset. The improvement becomes more significant for voxels near the object boundary, more than $40 \%$ for voxels with more than $12 \mathrm{~cm}$ radial offset. The improvement will increase if the object has nonuniformity, since the structures will produce more variation in the projection $\bar{y}_{i} / a_{i}^{2}$.

\section{B. Ideal PET System with NPW Observer}

For the ideal PET system described in the previous section, the SNR of the NPW observer for FBP reconstruction reduces to

$$
\mathrm{SNR}_{\mathrm{NPW}, \mathrm{FBP}}^{2} \approx \frac{1}{\xi_{j}^{2}} \frac{\left(\sum_{i} w_{i}^{2} \zeta_{i}^{2} \lambda_{i}^{2}\right)^{2}}{N \sum_{i} w_{i}^{4} \zeta_{i}^{2} \lambda_{i}^{3}}
$$

If we choose the filter to be

$$
w_{i}=\frac{1}{\lambda_{i}+\beta \mu_{i} / \kappa_{j}^{2}}
$$

(36) changes to

$$
\mathrm{SNR}_{\mathrm{NPW}, \mathrm{FBP}}^{2} \approx \frac{1}{\xi_{j}^{2}}\left(\sum_{i}\left(\frac{\lambda_{i} \zeta_{i}}{\lambda_{i}+\beta \mu_{i} / \kappa_{j}^{2}}\right)^{2}\right)^{2}\left(N \sum_{i} \frac{\lambda_{i}^{3} \zeta_{i}^{2}}{\left(\lambda_{i}+\beta \mu_{i} / \kappa_{j}^{2}\right)^{4}}\right)^{-1}
$$

Therefore, we can show that the improvement in lesion detectability with the NPW observer is also

$$
\frac{\mathrm{SNR}_{\mathrm{NPW}, \mathrm{MAP}}^{2}}{\mathrm{SNR}_{\mathrm{NPW}, \mathrm{FBP}}^{2}} \approx \kappa_{j}^{2} \xi_{j}^{2}>1
$$

This indicates that modeling the Poisson statistics in PET data should also improve human observer performance, as the NPW observer is correlated to human observers. 


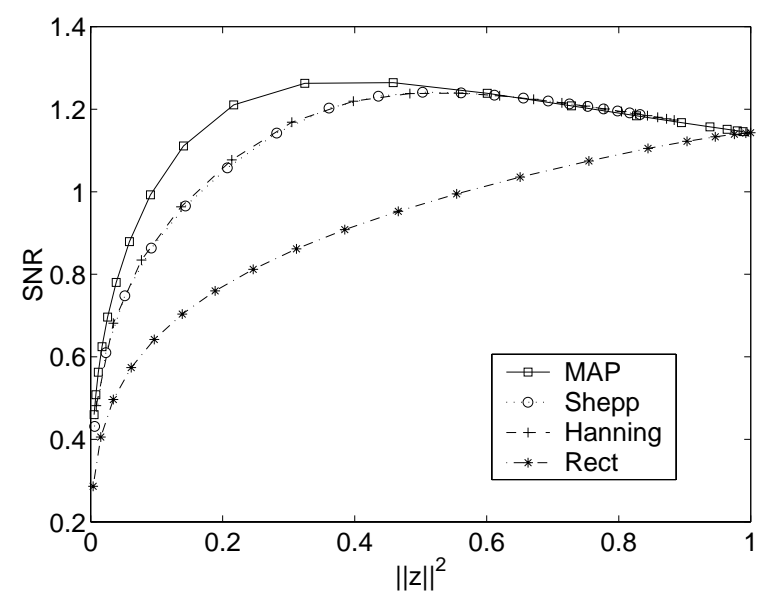

Fig. 3. $\mathrm{SNR}_{\mathrm{NPW}, \mathrm{FBP}}$ for detecting a point source lesion with different filters as functions of $\|\boldsymbol{z}\|^{2}$. All curves are plotted with $\xi_{j}=1$. "MAP" denotes the filter function in (37), "Shepp" the Shepp-Logan filter, "Hanning" the ramp filter with a Hanning window, and "rect" the ramp filter with a rectangular window.

\section{Effect of Filters on NPW Observer}

Eq. (39) is obtained by using (37) as the filter in the FBP algorithm. In practice, filters used in FBP reconstruction generally take the form of a ramp filter modified by a window function. Some commonly used filter functions are the ramp filter modified by a rectangular window (rect), the ramp filter modified by a Hanning window (Hanning), and the Shepp-Logan filter.

To examine the effect of the choice of filters on lesion detection, we plot in Fig. 3 the $\mathrm{SNR}_{\mathrm{NPW}}$,FBP for detecting a point source lesion with different filters as functions of $\|\boldsymbol{z}\|^{2}$. To some extent, $\|\boldsymbol{z}\|^{2}$ for a point source can act as a measure of resolution. ${ }^{3}$ For comparison, we also plot the $\mathrm{SNR}_{\mathrm{NPW} \text {,FBP }}$ with (37) as the filter function (denoted as "MAP" filter in Fig. 3). Clearly, the ramp filter with rectangular window has the worst performance. The filters with smooth window functions, Hanning and Shepp, are nearly the same. The MAP filter gives the best performance among all the filters studied. All the curves converge at the maximum resolution $(\|\boldsymbol{z}\|=1)$, where all filter functions become the ramp filter.

\section{Realistic PET System}

First, we study the PW observer. For a realistic system, $\boldsymbol{P}_{\text {sys }} \neq \boldsymbol{B}^{\prime}$, we have

$$
\frac{\mathrm{SNR}_{\mathrm{PW}, \mathrm{MAP}}^{2}}{\mathrm{SNR}_{\mathrm{PW}, \mathrm{FBP}}^{2}} \approx \kappa_{j}^{2} \xi_{j}^{2} \frac{\boldsymbol{f}_{l}^{\prime} \boldsymbol{P}_{\mathrm{sys}}^{\prime} \boldsymbol{P}_{\mathrm{sys}} \boldsymbol{f}_{l}}{\boldsymbol{f}_{l}^{\prime} \boldsymbol{P}_{\mathrm{sys}}^{\prime} \boldsymbol{B}^{\prime}\left(\boldsymbol{B} \boldsymbol{B}^{\prime}\right)^{-1} \boldsymbol{B} \boldsymbol{P}_{\mathrm{sys}} \boldsymbol{f}_{l}} .
$$

\footnotetext{
${ }^{3}$ If the local impulse response of a point source can be approximated by a Gaussian function, and the reconstruction preserves activity, the value of $\|\boldsymbol{z}\|^{2}$ is inversely proportional to the FWHM.
} 
Since $\boldsymbol{B}^{\prime}\left(\boldsymbol{B} \boldsymbol{B}^{\prime}\right)^{-1} \boldsymbol{B}$ is the projection operator onto the range space of $\boldsymbol{B}^{\prime}$, we have

$$
\frac{\boldsymbol{f}_{l}^{\prime} \boldsymbol{P}_{\mathrm{sys}}^{\prime} \boldsymbol{P}_{\mathrm{sys}} \boldsymbol{f}_{l}}{\boldsymbol{f}_{l}^{\prime} \boldsymbol{P}_{\mathrm{sys}}^{\prime} \boldsymbol{B}^{\prime}\left(\boldsymbol{B} \boldsymbol{B}^{\prime}\right)^{-1} \boldsymbol{B} \boldsymbol{P}_{\mathrm{sys}} \boldsymbol{f}_{l}} \geq 1
$$

where equality is achieved if and only if $\boldsymbol{P}_{\text {sys }} \boldsymbol{f}_{l} \in \operatorname{Range}\left(\boldsymbol{B}^{\prime}\right)$. However, in general, this condition is not satisfied due to depth-dependent sensitivity and detector blurring effects. This indicates that in addition to the benefit of modeling of the Poisson statistics of the data, MAP reconstruction can also improve the lesion detectability through accurately modeling the photon detection process of PET systems.

Next, we will prove similar results also hold for the NPW observer. We rewrite $\mathrm{SNR}_{\mathrm{NPW}}$,FBP for the realistic system as

$$
\mathrm{SNR}_{\mathrm{NPW}, \mathrm{FBP}}^{2} \approx \frac{1}{\xi_{j}^{2}} \frac{\left[\sum_{i} w_{i}^{2} \zeta_{i}^{2} \tilde{\lambda}_{i}^{2}(j)\right]^{2}}{N \sum_{i} w_{i}^{4} \tau_{i} \zeta_{i}^{2} \tilde{\lambda}_{i}^{2}(j)}
$$

To achieve the highest resolution, we set the filter to

$$
w_{i}=\frac{1}{\tilde{\lambda}_{i}}
$$

to deconvolve the blurring in $\boldsymbol{B} \boldsymbol{P}_{\mathrm{sys}}$. Then $\mathrm{SNR}_{\mathrm{NPW}, \mathrm{FBP}}$ changes to

$$
\mathrm{SNR}_{\mathrm{NPW}, \mathrm{FBP}}^{2} \approx \frac{1}{\xi_{j}^{2}} \frac{\left(\sum_{i} \zeta_{i}^{2}\right)^{2}}{N \sum_{i} \tau_{i} \zeta_{i}^{2} / \tilde{\lambda}_{i}^{2}(j)} .
$$

Similarly, we set $\beta=0$ in (25) and obtain the $\mathrm{SNR}_{\mathrm{NPW}, \mathrm{MAP}}$ of MAP reconstruction at the highest resolution,

$$
\mathrm{SNR}_{\mathrm{NPW}, \mathrm{MAP}}^{2} \approx \kappa_{j}^{2} \frac{\left(\sum_{i} \zeta_{i}^{2}\right)^{2}}{N \sum_{i} \zeta_{i}^{2} / \lambda_{i}(j)} .
$$

Again using the property that $\boldsymbol{B}^{\prime}\left[\boldsymbol{B} \boldsymbol{B}^{\prime}\right]^{-1} \boldsymbol{B}$ is the projection operator, we have

$$
\boldsymbol{f}^{\prime} \boldsymbol{P}_{\mathrm{sys}}^{\prime} \boldsymbol{P}_{\mathrm{sys}} \boldsymbol{f} \geq \boldsymbol{f}^{\prime} \boldsymbol{P}_{\mathrm{sys}}^{\prime} \boldsymbol{B}^{\prime}\left(B \boldsymbol{B}^{\prime}\right)^{-1} \boldsymbol{B} \boldsymbol{P}_{\mathrm{sys}} \boldsymbol{f}
$$

for any vector $f$. By setting $f$ to each Fourier transform basis function, we obtain

$$
\lambda_{i}(j) \geq \tilde{\lambda}_{i}^{2}(j) / \tau_{i}
$$

Therefore,

$$
\frac{\mathrm{SNR}_{\mathrm{NPW}, \mathrm{MAP}}^{2}}{\mathrm{SNR}_{\mathrm{NPW}, \mathrm{FBP}}^{2}} \geq \kappa_{j}^{2} \xi_{j}^{2},
$$

where equality is achieved if and only if $\boldsymbol{P}_{\text {sys }} \boldsymbol{f}_{l} \in \operatorname{Range}\left(\boldsymbol{B}^{\prime}\right)$. This proves that at the highest resolution MAP reconstruction is superior to FBP. The reconstructions obtained with other filters (for FBP) or $\beta$ value (for MAP) are equivalent to applying a filter to the highest resolution images. As indicated in Fig. 
3, the MAP-type filter is at least as good as the other filters commonly used with the FBP method, so we can expect MAP reconstruction to maintain higher SNR for lesion detection than FBP reconstruction at all resolution levels due to proper modeling of noise in the PET data and accurate modeling of the photon detection process.

\section{CONCLUSIONS AND Discussion}

We have theoretically studied lesion detectability of MAP reconstruction using the prewhitening and non-prewhitening observers and have compared the results to that of FBP reconstruction. The PW observer shows how much information is contained in reconstructed images, and the NPW observer partly predicts the performance of a human observer. We theoretically proved that the MAP algorithm can improve lesion detectability through accurately modeling the Poisson statistics in the data and the photon detection process in PET. Information for lesion detection is reduced in FBP reconstructions due to ignoring Poisson statistics and using the line integral model. With the PW observer and MAP reconstruction with quadratic smoothing priors, the ultimate lesion detectability is independent of the smoothing parameter and neighborhood. A nonquadratic prior and other prior information may further increase lesion detectability.

In this paper we used the SKE-BKE task and focused on detecting small lesion in the "low noise" situation. The analysis precludes the non-negativity constraints used in MAP reconstruction, but the result is a fair comparison to the regular FBP algorithm since FBP reconstruction may be improved by post processing using non-negativity constraints as well [37]. We note, however, the SKE-BKE task is a highly simplified scenario compared with real clinical detection tasks. Future work is needed to include randomness in both lesion and background.

In the analysis we have assumed that MAP reconstruction uses the exact system model because MAP reconstruction is amenable to arbitrary and complicated system models. However, in practice, the true system response is not exactly known, so there is always some error in the system model. If we consider such errors in the analysis, the performance of MAP reconstruction will be degraded by a factor shown in (41) with $\boldsymbol{B}$ equal to the projection matrix used in the MAP reconstruction. The degradation should be relatively small and insignificant, provided that the depth-dependent solid angle effect and detector response are properly modeled [23]. In this study we also assumed that the noise only comes from the Poisson statistics of the photon detection. In future studies we plan to include errors in normalization and attenuation correction factors.

As we have shown, proper detection of lesions relies heavily on the knowledge of the response to 
lesions, i.e., the observer template. In human observer studies such knowledge is acquired in the training process. As the FBP and MAP reconstructions both have spatially variant properties, the training process should include a large number of data sets. This is not a problem for FBP reconstruction, since most physicians are well trained to read FBP images. However, familiarizing physicians with MAP images is very important in human observer ROC studies; otherwise, results may be highly biased.

\section{REFERENCES}

[1] J. A. Fessler, "Penalized weighted least squares image reconstruction for PET," IEEE Transactions on Medical Imaging, vol. 13, pp. 290-300, 1994.

[2] E. Mumcuoglu, R. Leahy, S. Cherry, and Z. Zhou, "Fast gradient-based methods for Bayesian reconstruction of transmission and emission PET images,” IEEE Transactions on Medical Imaging, vol. 13, pp. 687-701, 1994.

[3] J. A. Fessler and A. O. Hero, "Penalized maximum-likelihood image reconstruction using space-alternating generalized EM algorithms,” IEEE Transactions on Image Processing, vol. 4, pp. 1417-1429, 1995.

[4] C. Bouman and K. Sauer, "A unified approach to statistical tomography using coordinate descent optimization," IEEE Transactions on Image Processing, vol. 5, pp. 480-492, 1996.

[5] L. Strauss and P. Conti, "The application of PET in clinical oncology," Journal of Nuclear Medicine, vol. 32, pp. 623-648, 1991.

[6] R. M. Leahy and J. Qi, "Statistical approaches in quantitative positron emission tomography," Statistics and Computing, vol. 10, pp. 147-165, 2000.

[7] D. R. Gilland, B. M. W. Tsui, C. E. Metz, R. J. Jaszczak, and J. R. Perry, "An evaluation of maximum likelihoodexpectation maximization reconstruction for SPECT by ROC analysis," Journal of Nuclear Medicine, vol. 33, pp. 451-457, 1992.

[8] J. Llacer, "Results of a clinical receiver operating characteristic study comparing filtered backprojection and maximum likelihood estimator images in FDG PET studies,” Journal of Nuclear Medicine, vol. 34, pp. 1198-1203, 1993.

[9] T. H. Farquhar, Improved Lesion Detection in Whole Body PET, Ph.D. thesis, University of California, Los Angeles, 1998.

[10] H. H. Barrett, "Objective assessment of image quality: Effects of quantum noise and object variability," Journal of the Optical Society of America A, vol. 7, pp. 1266-1278, 1990.

[11] H. H. Barrett, T. Gooley, K. Girodias, J. Rolland, T. White, and J. Yao, "Linear discriminants and image quality," Image and Vision Computing, vol. 10, pp. 451-460, 1992.

[12] J. Yao and H. H. Barrett, "Predicting human performance by a channelized Hotelling model," in Mathematical Methods in Medical Imaging, D. C. Wilson and J. N. Wilson, Eds., Proceedings of SPIE, vol. 1768, pp. 161-168, 1992.

[13] H. H. Barrett, J. Yao, J. Rolland, and K. Myers, "Model observers for assessment of image quality," Proc. Natl. Acad. Sci., vol. 90, pp. 9758-9765, 1993.

[14] S. C. Moore, D. J. deVries, B. Nandram, M. Kijewski, and S. Mueller, "Collimator optimization for lesion detection incorporating prior information about lesion size," Med. Phys., vol. 22, pp. 703-713, 1995.

[15] C. K. Abbey and H. H. Barrett, "Observer signal-to-noise ratios for the ML-EM algorithm," in Proceedings of SPIE, vol. 2712, pp. 47-58, 1996. 
[16] M. T. Chan, R. Leahy, and S. Cherry, "Comparing lesion detection performances of PET image reconstruction algorithms: a case study," IEEE Transactions on Nuclear Science, vol. 44, pp. 1558-1563, 1997.

[17] P. La Riviere, X. Pan, and B. Penney, "Ideal-observer analysis of lesion detectability in planar, conventional SPECT and dedicated SPECT scintimammography using effective multi-dimensional smoothing," IEEE Transactions on Nuclear Science, vol. 45, pp. 1273-1279, 1998.

[18] D. de Vries, M. King, E. Soares, B. Tsui, and C. Metz, "Effects of scatter subtraction on detection and quantitation in hepatic SPECT,” Journal of Nuclear Medicine, vol. 40, pp. 1011-1023, 1999.

[19] T. Narayan and G. Herman, "Prediction of human observer performance by numerical observers: an experimental study," Journal of Optical Society of America A, vol. 16, pp. 679-693, 1999.

[20] S. Wollenweber, B. Tsui, D. Lalush, E. Frey, K. LaCroix, and G. Gullberg, "Comparison of hotelling observer models and human observers in defect detection from myocardial SPECT imaging," IEEE Transactions on Nuclear Science, vol. 46, pp. 2098-2103, 1999.

[21] H. Gifford, M. King, D. de Vries, and E. Soares, "Channelized hotelling and human observer correlation for lesion detection in hepatic SPECT imaging," Journal of Nuclear Medicine, vol. 41, pp. 514-521, 2000.

[22] J. Qi, "Optimization of PET system design for lesion detection," in Proceedings of IEEE Nuclear Science Symposium and Medical Imaging Conference, Lyon, France, 2000.

[23] J. Qi, R. M. Leahy, S. R. Cherry, A. Chatziioannou, and T. H. Farquhar, "High resolution 3D Bayesian image reconstruction using the microPET small animal scanner," Physics in Medicine and Biology, vol. 43, pp. 1001-1013, 1998.

[24] J. Qi, R. M. Leahy, C. Hsu, T. H. Farquhar, and S. R. Cherry, "Fully 3D Bayesian image reconstruction for ECAT EXACT HR+," IEEE Transactions on Nuclear Science, vol. 45, pp. 1096-1103, 1998.

[25] R. D. Fiete, H. H. Barrett, W. E. Smith, and K. J. Myers, "Hotelling trace criterion and its correlation with human-observer performance," Journal of the Optical Society of America A, vol. 4, pp. 945-953, 1987.

[26] J. P. Rolland and H. H. Barrett, "Effect of random background inhomogeneity on observer detection performance," Journal of the Optical Society of America A, vol. 9, pp. 649-658, 1992.

[27] K. J. Myers, H. H. Barrett, M. C. Borgstrom, D. D. Patton, and G. W. Seeley, "Effect of noise correlation on detectability of disk signals in medical imaging," Journal of the Optical Society of America A, vol. 2, pp. 1752-1759, 1985.

[28] K. J. Myers and H. H. Barrett, “Addition of a channel mechanism to the ideal-observer model," Journal of the Optical Society of America A, vol. 4, pp. 2447-2457, 1987.

[29] J. Qi and R. M. Leahy, "Resolution and noise properties of MAP reconstruction for fully 3D PET," IEEE Transactions on Medical Imaging, vol. 19, pp. 493-506, 2000.

[30] J. Qi and R. M. Leahy, "A theoretical study of the contrast recovery and variance of MAP reconstructions from PET data," IEEE Transactions on Medical Imaging, vol. 18, pp. 293-305, 1999.

[31] J. Qi and R. M. Leahy, "Fast computation of the covariance of MAP reconstructions of PET images," in Proceedings of SPIE, vol. 3661, pp. 344-355, 1999.

[32] J. Fessler, "Mean and variance of implicitely defined biased estimators (such as penalized maximum likelihood): Applications to tomography," IEEE Transactions on Image Processing, vol. 5, pp. 493-506, 1996.

[33] J. A. Fessler and W. L. Rogers, "Spatial resolution properties of penalized-likelihood image reconstruction: Spatialinvariant tomographs," IEEE Transactions on Image Processing, vol. 9, pp. 1346-1358, 1996. 
[34] J. Pearl, "Asymptotic equivalence of spectral representations," IEEE Transactions on Acoustic, Speech and Signal Processing, vol. 23, pp. 547-551, 1975.

[35] R. E. Carson, Y. Yan, M. E. Daube-Witherspoon, N. Freedman, S. L. Bacharach, and P. Herscovitch, "An approximation formula for the variance of PET region-of-interest values," IEEE Transactions on Medical Imaging, vol. 12, pp. 240-250, 1993.

[36] R. Maitra and F. O'Sullivan, "Variability assessment in PET and related generalized deconvolution methods," Journal of American Statistical Association, vol. 93, pp. 1340-1355, 1998.

[37] F. O'Sullivan, Y. Pawitan, and D. Haynor, "Reducing negativity artifacts in emission tomography: post-processing filtered backprojection solutions," IEEE Transactions on Medical Imaging, vol. 12, pp. 653-663, 1993. 